\title{
Du corps jugé au corps désiré - le human enhancement en tant que processus historique
}

\section{Iris Ritzmann}

Medizinhistorisches Institut und Museum, Université Zurich
* Les références se trouveront sous www.bullmed.ch $\rightarrow$

Numéro actuel ou $\rightarrow$

Archives $\rightarrow 2013 \rightarrow 11$

Correspondance:

Académie Suisse des Sciences

Médicales (ASSM)

Petersplatz 13

CH-4051 Bâle

mail[at]samw.ch
Dans les débats autour de la classification normative des différentes interventions de human enhancement, on essaie souvent de définir des limites entre les interventions défendables et les interventions dommageables d'un point de vue médical, économiquement sûres et douteuses et finalement moralement admissibles et condamnables. Les avis actuels quant à l'emplacement de ces limites résultent de discours qui s'étendent sur des périodes prolongées et sont empreints de l'environnement socioculturel de l'époque.

C'est pourquoi le but de ce document n'est pas de faire un historique du human enhancement depuis l'antiquité jusqu'à nos jours. Il entend bien plus contribuer, à l'aide d'exemples ponctuels, à démontrer la relativité des avis actuels et, en même temps, à mettre en lumière les processus de développement par une étude de leur dimension historique.

L'histoire du human enhancement a été marquée par des processus significatifs à différents niveaux, qui ne peuvent être abordés que superficiellement dans ce document: au niveau social, la généralisation et l'établissement d'un modèle humain correspondant à une norme et à un idéal: au niveau économique, les transformations du marché médical et donc les modifications de la relation médecin-patient: et au niveau individuel les peurs et les attentes des patients dans un environnement orienté vers la perfection et le rendement.

\section{La médicalisation de la société depuis les «Lumières»}

Les traités historiques sur le human enhancement remontent souvent à l'antiquité, pour offrir ensuite au Moyen Age et à l'époque moderne des preuves que les êtres humains «have always been interested in creating or bettering themselves» [1]*. Plutôt que de chercher à élucider si cette attitude présente réellement une constance biologique et dans quelle mesure, l'analyse de la situation actuelle s'attache à étudier comment ces désirs de perfectionnement ont été appréhendés par les médecins, comment ils ont été discutés et instrumentalisés dans des milieux différents et quelle est leur portée.

Partant de ces questions, il est probable que l'analyse historique débute vers la fin du XVIII e siècle. C'est à cette époque que la médecine commence à s'intéresser aux désirs de perfectionnement de l'être humain. C'est également l'époque des prémisses d'un système de santé étatique qui définit la santé des individus dans le cadre de la société [2].
On entend par «Human Enhancement» les interventions médicales ou biotechnologiques dont l'objectif primaire n'est pas le traitement ou la prévention de maladies, mais I'«amélioration» de caractéristiques non pathologiques. Un groupe de travail des Académies Suisses des Sciences Médicales (ASSM) et des Sciences Humaines et Sociales (ASSH) a examiné minutieusement les questions éthiques soulevées par l'enhancement et publié ses analyses et recommandations dans une brochure intitulée «La médecine pour les personnes en bonne santé?» (www.academies-suisses.ch $\rightarrow$ Projets et thèmes). Des extraits de ce rapport paraissent à intervalles irréguliers dans le BMS.

Nous vivons aujourd'hui dans une société, dont les valeurs et les pratiques quotidiennes sont, dans une large mesure, empreintes de modes de pensée médicaux. Dans l'histoire de la médecine, ce phénomène est appelé «médicalisation». Les interprétations médicales interviennent dans nos choix relatifs à notre alimentation, à nos activités de loisirs, nos destinations de vacances. Mais ce «médecin intérieur», qui incarne la vision individuelle et unique de chaque individu, n'est pas seulement en étroite relation avec les expériences de la personne concernée: bien au contraire, il concerne également, plus largement, la compréhension sociale de la santé et donc de l'histoire de la médecine.

Au siècle des Lumières, des formations nationales paternalistes ont - pour des raisons politiques, militaires et économiques - focalisé leur attention sur la santé de leurs citoyens, une natalité aussi élevée que possible, des adultes travailleurs et féconds et la prévention de la pauvreté, des maladies et des handicaps. Le concept contemporain de «police médicale»connu grâce à l'ouvrage en six volumes «Système

\footnotetext{
Membres du groupe de travail

Prof. Dr méd. Dr phil. Nikola Biller-Andorno (présidente)

Prof. Dr méd. Anne-Françoise Allaz

Dr phil. Gaia Barazzetti (jusqu'en 2010)

lic. ès lettres Nadja Birbaumer, ASSH

PhD Susanne Brauer, NEK-CNE

Prof. Dr méd. Jürg Kesselring

Prof. Dr méd. lic. phil. Iris Ritzmann

Dr phil. Simone Romagnoli

Dr biol. Adrian Rüegsegger, TA-SWISS

lic. iur. Michelle Salathé, MAE, ASSM

PD Dr théol. Markus Zimmermann-Acklin
} 
d'une police médicale complète» publié par Johann Peter Frank de 1779 à 1819 - définit une idée de la protection de la santé publique, qui, au moyen de règles, intervient dans le comportement de la population et accompagne les humains «du berceau à la tombe».

L'activité médicale au XVIII ${ }^{\mathrm{e}}$ siècle est en étroite relation avec les efforts déployés par les médecins pour améliorer la «santé de la population». La notion de «médecine des Lumières» est caractérisée par la création d'institutions de prévoyance et la lutte contre les guérisseurs non habilités, mais également par l'activité publicitaire croissante de certains médecins. La santé est devenue un bien social qu'il convient de protéger et de conserver.

Les guides de santé populaires ont connu un essor sans précédent: les ouvrages médicaux étaient adressés, dans la langue nationale, à un large public, également aux habitants des zones rurales ou éloignées, dans la mesure où ceux-ci savaient lire. Dans leurs titres et préfaces, les auteurs mettaient clairement en avant que leur objectif était d'instruire et d'informer la population sur les thèmes de la santé et de la maladie. Dans la deuxième moitié du XVIII ${ }^{e}$ siècle, de nombreuses revues abordaient des questions médicales et d'éducation sanitaire. Avec une part croissante de lecteurs au sein de la population, certains médecins - entre autres de nombreux piétistes - ont réussi à diffuser et à consolider leurs conceptions de la santé grâce à la presse.

\section{La profession de médecin entre service et autorité professionnelle}

Dans son rapport d'experts «Buts et missions de la médecine au début du $21^{\text {e }}$ siècle», l'ASSM affirme que le «respect de la dignité humaine et le respect de l'autodétermination du patient sont à la base de tout acte médical» [3]. Même si l'évolution tend à s'inverser, la relation médecin-patient reste encore souvent caractérisée, du côté du médecin, par la connaissance et le pouvoir et, du côté du patient, par la dépendance. La responsabilité des patients devrait donc être encouragée, ce qui soulève toutefois des résistances de part et d'autre. Dans quelle mesure les patients ont-ils le droit de poser des exigences et d'utiliser l'offre de soins à leur guise? Répondre à ces questions implique aussi une réflexion sur les interventions de human enhancement actuellement proposées: les aspects financiers et, tout à fait fondamentalement, la compréhension des rôles du médecin et du patient doivent être analysés.

Les médecins de l'époque moderne antérieure n'avaient pas encore le monopole du marché médical. Ils étaient obligés de défendre leur position par rapport aux autres guérisseurs traditionnels et libéraux et se trouvaient dans un état de dépendance financière maximale par rapport aux patients. Comme en témoignent les guides de santé du XVIIIe siècle, le médecin devait gagner des clients réguliers qui se tournent vers lui avec confiance, qu'ils soient ma- lades ou en bonne santé. Tout à fait indépendamment de la qualité thérapeutique, il s'agissait de persuader la clientèle que lui - et lui seul - sera à même de les maintenir en bonne santé ou de leur permettre de recouvrer leur santé.

Au XVIII' ${ }^{e}$ siècle, la clientèle régulière du médecin érudit était composée d'un groupe gérable de patients privés, et le revenu du médecin dépendait finalement de leur disposition à le rétribuer. L'historiographie médicale désigne cette forme de relation entre le médecin et le patient par «système de patronage». * Notamment aux XVII et XVIII siècles, le patient endossait un rôle de patron, dont le médecin diplômé briguait les faveurs. Même si certains médecins étaient financièrement indépendants, la grande majorité d'entre eux devait s'efforcer d'assurer leur existence dans un tel marché.

Ainsi, le fait de se distinguer des autres prestataires était tout à fait essentiel pour l'activité médicale. Ce n'est qu'au courant du XIX ${ }^{\mathrm{e}}$ siècle que les médecins académiques ont acquis un certain monopole du marché médical et, par là même, un certain pouvoir de définition des notions telles que santé et maladie, dangereux et sans risque, efficace et obsolète. Ils se sont de plus en plus imposés pour décider quelles activités thérapeutiques devaient être reconnues et quelles pratiques les autorités devaient interdire, nota bene sans pour autant être plus à même de guérir que leurs concurrents. La pluralité de l'offre diminuait à vue d'œil et le sérieux des guérisseurs non académiques était mis en doute. Parallèlement, la confiance envers les médecins diplômés a grandi, une confiance qui, jusqu'à ce jour, constitue la base de la relation médecin-patient et inhibe l'autonomie des malades.

Cette promotion du corps médical académique est allée de pair avec l'instauration d'un nouveau système de santé. Bien plus que les thérapies disponibles, c'est la médicalisation croissante de la société qui a amorcé cette tendance. Dans ce contexte, les médecins peuvent se situer en tant qu'experts chargés de déterminer quels dangers menacent la population et de trouver les moyens de les combattre. Les enseignements en sciences naturelles ont également apporté aux médecins académiques une base argumentaire qui, au lieu d'opposer leurs propres concepts à ceux des autres, défend une science objective et indépendante.

\section{La santé collectivisée}

La relation personnelle médecin-patient s'est transformée avec l'apparition des missions collectives de la médecine. Les médecins n'étaient plus seulement chargés de patients individuels, mais également de la santé de l'ensemble de la population. Protéger la «santé publique» des dangers potentiels était devenu une mission de la médecine.

Egalement le patient devenait responsable de sa propre santé et, au-delà, de la santé de toute la population. La santé était devenue la condition nécessaire 
à une vie consacrée au travail et à la reproduction au niveau de l'individu, mais également à la défense, à la capacité économique et au bien-être de toute la «nation».

La médecine préventive a pu évoluer grâce aux efforts déployés pour éloigner les menaces pour la santé: ses offres s'adressent en priorité aux personnes en bonne santé, tant au niveau large de la société qu'au niveau micro de l'individu. Certes, les scénarios des risques médicaux doivent être diffusés explicitement avant de bénéficier de la reconnaissance générale. Ces stratégies ne s'expliquent pas simplement sur la base de faits historiques. Après l'acceptation des théories de Ludwik Fleck, l'idée s'est imposée qu'il n'existe aujourd'hui ni «la» médecine - en tant que système uniforme, en constante évolution et logique en soi - ni une science indépendante. dies en catégories séparées. C'est justement cette classification des maladies qui implique l'observation de normes.

Le diagnostic et la thérapie, mais aussi plus généralement la santé et la maladie ont perdu leur individualité. La perception du corps a été modifiée par le thermomètre, la prise du pouls, la fréquence respiratoire et finalement la visualisation des activités organiques électriques. Ce n'était plus ceux qui se sentaient en bonne santé qui l'étaient, mais ceux dont la température du corps s'élevait entre 35,5 et $37^{\circ} \mathrm{C}$. La visualisation de l'intérieur du corps - radiologie, échographie, TC et IRM - permettait de détecter avec de plus en plus de précisions des nouveaux écarts par rapport à la norme. Grâce aux radiographies, le degré de gravité d'une infection pulmonaire pouvait être défini: les échographies fournissaient des indications

\section{L'établissement d'une culture médicale d'expert a influencé aussi bien l'offre thérapeutique que la perception de la corporalité.}

Les médecins définissaient ce qu'englobait la notion de santé, quels dangers la menaçaient et comment y pallier. Par exemple, les médecins du XVIII siècle identifiaient l'onanisme comme un mal terrible qui menaçait toute la jeune génération. Au $\mathrm{XIX}^{\mathrm{e}}$ siècle, ils reconnurent dans la chute de la natalité un nouveau danger qu'il fallait écarter. Dans les années 1880, l'alcoolisme et la criminalité étaient dans la ligne de mire des médecins, et quelques décennies plus tard, la menace d'une dégénérescence de la population civile devait être éradiquée par des mesures eugéniques. La «science» de l'hérédité et des races, considérée à cette époque comme moderne et d'avenir, était propagée par les médecins de tous les pays occidentaux et enseignait que les porteurs de gènes étrangers menaçaient la santé et la vie de toute la population. La volonté de rendre stérile et même d'éliminer les individus prétendument hors normes était principalement basée sur la peur de la dégénérescence et de la perdition.

\section{Normalisation de la santé et de la maladie}

L'établissement d'une culture médicale d'expert a influencé aussi bien l'offre thérapeutique que la perception de la corporalité. Des critères mesurables permettant de différencier la maladie de la santé et d'établir des directives générales sont indispensables à un système moderne chargé de la santé d'une population croissante. L'enseignement antique initial de la pathologie humorale, selon lequel l'équilibre individuel entre les différents fluides était responsable de la santé et chaque maladie et pouvait être expliquée par la personnalité du patient et ses habitudes, a fait place à une systématisation visant à classer les mala- quant à la normalité d'une grossesse et de l'enfant à naître, ou encore une IRM révélait une transformation jusque-là ignorée.

L'imagerie, les valeurs des tableaux, les analyses du sang et des gènes ont fait apparaître une nouvelle catégorie de patients, n'étant ni malades, ni conformes aux normes. Les possibilités diagnostiques de plus en plus techniques ont réduit la part d'individus «normaux». Un nombre croissant de personnes devenues des patients potentiels, par exemple en raison d'une baisse des valeurs normatives de la pression artérielle ou d'analyses génétiques, se sont senties menacées au quotidien par la maladie et la mort et sont devenues des consommateurs réguliers des prestations médicales.

Les représentations normatives du corps ne dominaient pas seulement le discours médical spécialisé: elles ont également largement influencé la perception de la maladie et de la santé d'une grande partie des citoyens. Dans une société de plus en plus orientée vers le collectif, l'orientation selon des normes a gagné en importance - le «médecin intérieur» s'insinue de plus en plus dans le comportement au quotidien.

\section{L'enhancement sous le signe de normes idéalisées}

La norme ne correspond pas simplement à une partie définie d'une distribution gaussienne. La norme, c'est aussi être jeune, beau et viril, comme le révèlent par exemple les manuels d'anatomie et autres témoignages visuels de la compréhension du corps. Une peau normale et donc «saine» est, jusque dans nos conceptions actuelles, une peau sans ride ni taches, 
un ventre normal est plat, un nez normal est droit et des dents normales sont blanches et régulières. Egalement le poids normal et sain ne correspondait plus au poids moyen, mais a gagné sa propre dynamique, de telle façon que la norme elle-même a soudain commencé à devenir anormale, malsaine et pathologique. Cette norme transformée ne représentait pas seulement la santé, mais devenait un idéal.

Si l'on voulait correspondre à cette norme idéalisée, c'est-à-dire être perçu comme normal et sain, le corps et l'esprit devaient être modelés en conséquence. Les médecins ont - du moins en partie - influencé ces demandes d'ajustement du moi à des conceptions idéales et l'éventail des offres correspondantes tend à s'élargir de plus en plus. L'optimisation humaine est devenue un secteur économique attractif.

Au niveau individuel, on a pu observer comment les médecins ont essayé d'élargir la palette de leurs prestations thérapeutiques avec des offres d'enhancement, tout en luttant en même temps vigoureusement contre les offres similaires émanant de concurrents non-médecins [5]. A titre d'exemple, dans des publications médicales du XVIII ${ }^{\mathrm{e}}$ siècle, les agents éclaircissants pour le teint étaient présentés en même temps comme prescriptions utiles que comme charlatanerie mortelle. Egalement des ouvrages comme «Makrobiotik oder die Kunst, das menschliche Leben zu verlängern» (L'art de prolonger la vie par la macrobiotique) de Christoph Wilhelm Hufeland paru en 1797, témoignent finalement d'une compréhension des missions médicales qui ne se limite pas à soulager et à guérir, mais répond également à des demandes telles que prolonger la vie, rajeunir le corps, embellir l'aspect ou améliorer les capacités.

\section{Le $\mathrm{XIX}^{\mathrm{e}}$ siècle vit émerger des médicaments contre les «dangers de l'obésité», les maladies des nerfs, les faiblesses ou la fatigue.}

Le XIX $^{\mathrm{e}}$ siècle vit émerger des médicaments contre les «dangers de l'obésité», les maladies des nerfs, les faiblesses ou la fatigue. Les correcteurs de hanches, de tête et de nez ont été vantés dans les revues. Dans la première moitié du $\mathrm{XX}^{\mathrm{e}}$ siècle, ces produits se vendaient comme des petits pains, surtout lorsqu'ils permettaient de cacher des caractéristiques de races prétendument inférieures. Non seulement la forme extérieure devait correspondre à l'idéal, mais également les performances devaient atteindre un niveau maximal idéal dans le contexte de l'industrialisation: ceci concernait en priorité les hommes.

A l'aide d'extraits de testicules, des médecins ont essayé d'augmenter la «force de vie» et ont déve- loppé, au début du XX $\mathrm{X}^{\mathrm{e}}$ siècle, des cures de jouvence en s'appuyant sur les nouvelles expérimentations des domaines de l'endocrinologie et de la médecine de transplantation [6]. Même si ces médecins devaient marcher sur la corde raide pour éviter d'être considérés comme des charlatans, ils finissaient toujours par remporter l'approbation de médecins académiques de haute réputation. La thérapie par les cellules fraîches, développée dans les années 1930 par le chirurgien suisse Paul Niehans, a été suivie dans les milieux aisés, mais - fait révélateur - seulement dans l'après-guerre. Même si ce n'était que provisoirement, Niehans a acquis une telle renommée qu'il avait été intégré à l'Académie pontificale des sciences - en tant que successeur d'Alexander Fleming qui avait découvert la pénicilline [7].

Mais où s'arrête la médecine comme activité curative ou lénifiante? Quand devient-elle une offre de marché qui prend en compte les besoins d'une clientèle ne pouvant être définie comme des patients? Cette question se pose encore et toujours, qu'il s'agisse de la thérapie de cellules fraîches de Niehans ou de la prescription de Viagra [8].

Les médecins ont pour mission de soigner et soulager les souffrances physiques ou psychiques, également de les éviter à l'aide de la médecine préventive. Dans le contexte de la médicalisation, les écarts par rapport à la norme apparaissent de plus en plus comme des dangers susceptibles d'engendrer des souffrances physiques ou psychiques: dès lors, le soulagement de ces souffrances fait également partie des missions de la médecine. Ces dangers comportent des risques physiques comme une morbidité accrue et une espérance de vie diminuée. Mais ils incluent également des risques psychiques tels que l'exclusion et l'isolement. En conséquence, les efforts déployés pour améliorer sa personne, aussi bien physiquement que psychiquement, sont en relation directe avec le désir de se libérer de sa souffrance ou d'écarter les possibles menaces. Dans cette interprétation, les offres d'enhancement en médecine servaient à soulager et éviter les souffrances au même titre que les offres thérapeutiques classiques supposées sérieuses. Il est donc difficile de mettre en place des limites claires et nettes.

\section{L'intériorisation des idéaux}

Mais le contre-modèle d'un idéal performant, sportif, jeune et svelte n'est pas simplement apparu du fait d'un pouvoir extérieur capable d'influencer les valeurs sociales. Il correspondait à une morale qui prédominait, plus ou moins fortement, dans toute la population. Par la propagation médicale, cette morale acquit toutefois un potentiel de persuasion supplémentaire et a pu être d'autant mieux acceptée par les individus dans le sens de techniques du soi $[9,10,11]$. Ainsi, audelà des représentations individuelles de la santé, les pratiques isolées reflètent l'approche socioculturelle des écarts par rapport aux normes [12]. 
Les représentations de la santé et de la beauté se transforment aussi au gré de la conjoncture. A l'époque de l'essor économique, des mannequins comme Twiggy représentaient une image fragile et infantile de la femme. Dans les années 1990, le corps idéal de la femme devait être jeune, musclé, entraîné et performant. L'homme paternel et protecteur avait perdu son prestige à cette époque, les abondantes chevelures disparurent et les muscles s'exhibaient sous une peau rasée de près. En même temps, les centres de fitness connurent un essor sans précédent et la consommation d'anabolisants n'était plus réservée aux sports d'élite. Pour citer un autre exemple, la consommation d'héroïne, drogue sédative, a de plus en plus reculé devant d'autres drogues stimulantes, comme la cocaïne, compatibles avec une activité professionnelle «normale».

Avec l'intériorisation des valeurs sociales, il devient difficile d'évaluer les désirs individuels de chaque personne. Il se peut qu'une personne ne veuille subir une intervention d'amélioration que parce qu'elle est guidée par des représentations extérieures. Ou cette «influence» des discours ne doit-elle pas aussi être constamment considérée comme une interprétation active personnelle, qui conditionne finalement la conviction personnelle [13] diminuer la souffrance individuelle. Leur évaluation doit tenir compte de cette question problématique et non pas la réprouver d'emblée.

\section{Conclusion}

- L'analyse historique confirme que la maladie doit être comprise comme un concept socioculturel, orienté selon l'intérêt. Il est impossible de tracer des limites claires et «objectives» entre la thérapie et la prévention d'une part, et l'enhancement d'autre part. Si l'évolution historique détermine ce qui doit être compris comme menace pour la santé, elle a toutefois montré que certaines conceptions pour préserver ou pour maximiser la santé peuvent avoir des conséquences fatales pour l'individu concerné ainsi que pour la société.

- De même, ce qui est entendu comme norme (de santé) pertinente évolue constamment; le corps médical participe aussi activement à ce processus. Dès que les représentations des normes définies et idéalisées par la société sont intériorisées, il est difficile de les distinguer des préférences personnelles de la personne. Des écarts par rapport à la représentation idéale peuvent être à l'origine de souffrances profondes.

\section{En règle générale, une demande d'enhancement correspond à un désir de reconnaissance sociale.}

Comment l'individualité peut-elle être préservée compte tenu de la pression collective sur l'apparence extérieure? Dans les pays riches et surtout dans les milieux fortunés, les représentations normatives peuvent être réalisées grâce à des interventions médicales. Des dents blanches et régulières, des visages sans rides, des poitrines fermes, des bras musclés et des tailles minces ne témoignent pas seulement de la «beauté» et de la performance, mais de plus en plus aussi, dans le sens d'une distinction, de l'appartenance à un milieu aisé. De ce fait, la question de l'accessibilité aux offres de human enhancement doit impérativement englober cette dimension sociopolitique et socio-éthique.

En règle générale, une demande d'enhancement correspond à un désir de reconnaissance sociale. Les efforts déployés pour éclaircir une peau foncée, pour redresser un nez tordu ou pour éliminer la graisse témoignent de la tendance à s'orienter selon les valeurs sociales en vigueur. Même si cette tendance cache manifestement une hiérarchisation et une discrimination d'individus sur la base de leur apparence extérieure - qualifiée aujourd'hui de «lookisme»-, il n'empêche que finalement ces pratiques servent à
- Avec la médicalisation croissante, les médecins sont devenus responsables d'aspects concernant le quotidien (alimentation, apparence, habitudes de vie, etc.). Ce n'est que depuis le XIX ${ }^{\mathrm{e}}$ siècle que la médecine propose des interventions d'enhancement et exige, de ce fait, des expertises dans ce domaine. Les tentatives visant à distinguer strictement la médecine véritable et «sérieuse» des pratiques d'amélioration orientées au marché, sont, elles aussi, parfois empreintes d'intérêts économiques et politiques.

- La compétence et l'expertise des médecins, qui résultent de l'évolution historique et qui touchent

de nombreux domaines de la vie quotidienne, impliquent également une lourde responsabilité. Ainsi, les décisions cliniques doivent non seulement prendre en compte le patient individuel, mais également réfléchir aux conséquences sur le bien-être

public, tant au regard des budgets publics limités que des évolutions sociales problématiques (par ex. exigences exacerbées au niveau du rendement ou de la disponibilité). 


\section{Références}

1 Wiesing U. The History of Medical Enhancement: From Restitutio ad Integrum to Transformatio ad Optimum? In: Gordjin B, Chadwick R (eds.). Medical Enhancement and Posthumanity. The International Library of Ethics, Law and Technology 2. Heidelberg: Springer; 2008: 9-24, ici 9.

2 Wahrig B, Sohn W (Hrsg.). Zwischen Aufklärung, Policey und Verwaltung: Zur Genese des Medizinalwesens 1750-1850. Wiesbaden: Harrassowitz; 2003.

2 Académie Suisse des Sciences Médicales (ASSM). Buts et missions de la médecine au 21e siècle. Bâle: 2004.

4 Stolberg M. Die Harnschau: Eine Kultur- und Alltagsgeschichte. Böhlau: Köln; 2009: v. a. 213-23.

5 Sander S. Der Arzt und die Kosmetik - Zerrbilder im Spiegel der deutschen Medizinhistoriographie. Medizinhist J. 2002;37: 265-300.

6 Schlich T. Die Erfindung der Organtransplantation: Erfolg und Scheitern des chirurgischen Organersatzes 1880-1930. Frankfurt a. M.: Campus; 1998, v.a. 153-77.

7 Hofer HG. Frischzellen-Fama: Paul Niehans und die westdeutsche Aufbaugesellschaft der 1950er Jahre. In: Eschenbruch N, Balz V, Klöppel U, Hulverscheidt M (Hrsg.). Arzneimittel des 20. Jahrhunderts: Historische Skizzen von Lebertran bis Contergan. Science Studies. Bielefeld: Transcript; 2009: 229-53.
8 Werner MH. Viagra - rechtliche und ethische Fragen. Ärzteblatt Baden-Württemberg. 2002; 81(10): 1-4.

9 Möhring M. Die Regierung der Körper «Gouvernementalität» und «Techniken des Selbst»: Zeithistorische Forschungen/Studies in Contemporary History. Online-Ausgabe. 2006; 2: 3.

10 Maasen S. Schönheitschirurgie: Schnittflächen flexiblen Selbstmanagements. In: Orland B (Hrsg.) Artifizielle Körper. Zürich: Chronos; 2005: 239-59

11 Lengwiler M, Madarász J (Hrsg.). Das präventive Selbst: Eine Kulturgeschichte moderner Gesundheitspolitik. Bielefeld: Transcript; 2010.

12 Gilman S. Making the Body Beautiful: A Cultural History of Aesthetic Surgery. Princeton: Princeton University Press; 1999.

13 Villa P-I. Wider die Rede vom Äusserlichen. In: Villa P-I (Hrsg.). Schön normal: Manipulationen als Technologien des Selbst. Bielefeld: transcript; 2008: 7-18. 